\title{
Research on a Remote Sensing Image Classification Algorithm Based on Decision Table
}

\author{
Dehui Zhang ${ }^{1, a}$, Yong Yang ${ }^{1, b}$, Kai Song ${ }^{2, c}$, Deyu Zhang ${ }^{2, d}$ \\ ${ }^{1}$ School of Information and Electrical Engineering, Shenyang Agricultural University, Shenyang, \\ 110161, China \\ ${ }^{2}$ School of Information Science and Engineering, Shenyang Ligong University, Shenyang, 110159, \\ China \\ aemail: zdh_syit@163.com, bemail: yy_syau@163.com, 'email: songkai_syit@163.com, ${ }^{d}$ email: \\ zhang_de_yu@163.com
}

Keywords: High Resolution Satellite Remote Sensing Image; Decision Table; Feature Attribute; Object Oriented

\begin{abstract}
A remote sensing image classification algorithm based on decision table is proposed in this study. Firstly, image segmentation of remote sensing image was performed to extract feature attribute of image object. Then several samples were selected from image objects in different categories to construct classification information system. Thereafter, classification attributes were discretized to build decision table and obtain classification rules. Finally, classification of samples was realized by rule matching. Our experiment shows that this novel algorithm can classify earth objects in high resolution satellite remote sensing image, and present the actual distribution of earth objects.
\end{abstract}

\section{Introduction}

Recognition of earth object by using high resolution satellite remote sensing image is one of the most important applications of remote sensing techniques [1]. Moreover, remote sensing classification techniques is essential for dynamic monitoring of change information, thematic information extraction, thematic mapping, construction of remote sensing database from remote sensing images [2].

In the early years, remote sensing image classification techniques recognize and analyze type of earth objects by manual interpretation of elements and markers, which largely depends on knowledges and experiences of the interpreter. This manual method can be hardly used in processing massive remote sensing image data [3,4]. In recent years, a number of classification methods have been developed, including neural network, expert system, support vector machine, etc. [5]. Although these methods are much better than the traditional ones, they still have some limitations. For example, due to the category complexity and irregular distribution of earth object in high resolution remote sensing image, it is difficult to obtain classification of earth object from massive amount of remote sensing image [6]. To resolve these problems, we propose a remote sensing image classification algorithm based on decision table by taking the advantages of high resolution satellite remote sensing image.

\section{Basic Ideas of Algorithm}

The basic ideas of the remote sensing image classification algorithm based on decision table are as following:

Step 1. Establishment of classification information system

By utilizing the features of the investigated areas, sample objects of various earth objects are selected to establish classification information system.

Step 2. Discretization of the classification attributes

The texture features are extracted from the image object, which is further discretized by attribute 
discretization methods.

Step 3. Construction of decision table

The selected sample objects from various earth objects from step 1 are used to construct decision table.

Step 4. Simplifying decision table

The simplified decision table is build up by attribute reduction.

Step 5. Obtaining the rules

The confidence level of the rule between equivalence class of condition attribute and decision attribute is calculated. If the confidence level is 1 , the rule is formed.

Step 6. Obtaining the preliminary classification result of remote sensing image

According to the mean spectrum features, the resulting image objects are classified in the most reasonable and optimal way to get the preliminary classification results.

Step 7. Obtaining the final classification result of remote sensing image

After getting the preliminary classification result of remote sensing image, texture attribute of all the non-sample objects except for the selected sample objects in step 1 are discretized. Then matching of discretized texture attribute with classification rule in step 5 is performed to obtain the final classification result of remote sensing image.

\section{Implementation of Algorithm}

\subsection{Establishment of the classification information system}

By using object oriented multiresolution segmentation method, segmentation map composed of image object are obtained. Then feature attribute is extracted from image object and regarded as condition attribute; several samples are selected from different earth objects to build up construction attribute. Finally, classification information system is constructed according to both condition attribute and decision attribute.

\subsection{Discretization of the classification attribute}

From segmentation map of remote sensing image, the texture feature is extracted and further discretized by using attribute discretization method.

Calculating sing attribute discretization model:

Len(Model) + Len(Data $\mid$ Model)

Corresponding objective function of the above model:

$\operatorname{SAD}\left(\mathrm{t}_{\mathrm{i}}, \mathrm{t}_{\mathrm{i}+1}\right)=\mathrm{F}_{1}\left(\mathrm{t}_{\mathrm{i}}, \mathrm{t}_{\mathrm{i}+1}\right)+\mathrm{F}_{2}\left(\mathrm{t}_{\mathrm{i}}, \mathrm{t}_{\mathrm{i}+1}\right)$

In (2),

$$
\begin{aligned}
& \mathrm{F}_{1}\left(\mathrm{t}_{\mathrm{i}}, \mathrm{t}_{\mathrm{i}+1}\right) \approx-\log P(\text { Model }) \\
& P(\text { Model })=\mathrm{P}\left(\left|\mathrm{T}_{\mathrm{k}}\right|=\delta_{\mathrm{k}},\left\{\mathrm{C}_{\mathrm{k}}=\mathrm{S}_{\mathrm{k}}\right\},|\mathrm{T}|=M,\left\{T_{k j}=M_{k j}\right\}\right)
\end{aligned}
$$

From (3) and (4),

$$
\left.\mathrm{F}_{1}\left(\mathrm{t}_{\mathrm{i}}, \mathrm{t}_{\mathrm{i}+1}\right) \approx \log (\mathrm{M}-1)+\log C_{\delta_{k-1}}^{S_{k-1}}+\log C_{S}^{S_{k}}+\log (\mathrm{N}-1)+\sum_{k=i}^{i+1} \log C_{\delta_{k}+s_{k}-1}^{S_{k-1}}\right)
$$

\subsection{Establishment of decision table}

Each row in the decision table records two information of the image object. One is the feature attribute, and the other is the class attribute of the image object. As shown in Table 1, mean of the spectrum feature is the feature attribute of the image object, while body of water, building and vegetation is the class attribute of the image object. Specifically, 1 represents for body of water, 2 for building and 3 for vegetation in this table. 
Table 1 Classification decision table

\begin{tabular}{ccccc} 
Sample & $\begin{array}{c}\text { Grayscale value of } \\
\text { red band }\end{array}$ & $\begin{array}{c}\text { Grayscale value of } \\
\text { green band }\end{array}$ & $\begin{array}{c}\text { Grayscale value of } \\
\text { blue band }\end{array}$ & Classification \\
\hline 1 & 33.25 & 28.76 & 40.16 & 1 \\
2 & 166.25 & 132.11 & 142.54 & 2 \\
3 & 42.15 & 45.78 & 42.93 & 2 \\
4 & 122.36 & 98.76 & 99.32 & 2 \\
5 & 87.56 & 112.43 & 85.76 & 3 \\
6 & 102.75 & 100.12 & 101.99 & 3 \\
7 & 81.55 & 130.76 & 87.43 & 3 \\
8 & 70.32 & 107.32 & 76.97 & 3 \\
9 & 102.12 & 148.65 & 109.25 & 3 \\
10 & 98.77 & 134.65 & 100.21 & 3 \\
\hline
\end{tabular}

Although one image object has many feature attribute (spectrum attribute, texture attribute, etc.), not each of them is related to classification of remote sensing image. On the condition that the classification function of decision is not affected, we can delete some irrelevant attributes to simplify decision table [7, 8]. The details of simplification are as the following:

(1) Each sample object has condition attribute $\mathrm{C}$ and decision attribute $\mathrm{D}$.

(2) If a certain condition attribute $\mathrm{c}$ is to be deleted from the condition attribute set $\mathrm{C}$, two formulas needs to be calculated. One is dependency formula for calculating dependency degree of decision attribute $\mathrm{D}$ on condition attribute $\mathrm{C}$. The other is importance formula for calculating importance of condition attribute $\mathrm{C}$ to decision attribute $\mathrm{D}$. By calculating the two formulas, whether the certain condition attribute $\mathrm{c}$ relative to decision attribute $\mathrm{D}$ should be reduced can be determined.

If $S G F(c, C, D) \neq 0$, then condition c relative to $D$ cannot be reduced;

If $\operatorname{SGF}(\mathrm{c}, \mathrm{C}, \mathrm{D})=0$, condition c relative to $\mathrm{D}$ can be reduced.

(3) Calculating importance of condition attribute to decision attribute:

$\operatorname{SGF}(c, C, D)=\gamma(C, D)-\gamma(C-\{c\}, D)$

SGF(c, C, D) denotes proportion of the incorrectly classified samples during deletion of certain attribute c from attribute set.

(4) Calculating dependency degree of decision attribute D on condition attribute C:

$\gamma(\mathrm{C}, \mathrm{D})=|\mathrm{POS}(\mathrm{C}, \mathrm{D})| /|\mathrm{U}|$

In (7),

$\gamma(\mathrm{C}, \mathrm{D})$ denotes dependency degree of decision attribute $\mathrm{D}$ on condition attribute C;

$\gamma(C-\{c\}, D)$ denotes dependency degree of decision attribute $D$ on condition attribute $C$ with certain condition attribute $\mathrm{c}$ being deleted;

POS(C, D) denotes a set, and elements in this set include the set of the image object which is correctly classified in to the decision attribute D;

$\mathrm{U}$ denotes a set, and elements in this set include all the image objects appeared in the segmentation map;

|| denotes number of the data elements in the set.

4. Classification result of the remote sensing image

According to the extracted mean of spectrum, the image objects are classified in the most reasonable and optimal way to get the preliminary classification results.

In the preliminary classification results, texture attribute of all the non-sample objects from 
different classes are discretized. Then matching of discretized texture attribute with classification rule is performed to obtain the final classification result of remote sensing image.

By using object oriented multiresolution segmentation method, segmentation map of remote sensing image are obtained. In the map, the selected sample objects are labeled with white circles. The segmentation map of remote sensing image of selected sample objects is shown in Fig. 1 . The preliminary classification result of remote sensing image is shown in Fig. 2 (1 represents for building, 2 for vegetation and 3 for water surface). The final classification result of remote sensing image is shown in Fig. 3 (1 represents for building, 2 for shadow, 3 for woodland, 4 for farmland, 5 for grassland and 6 for water surface).

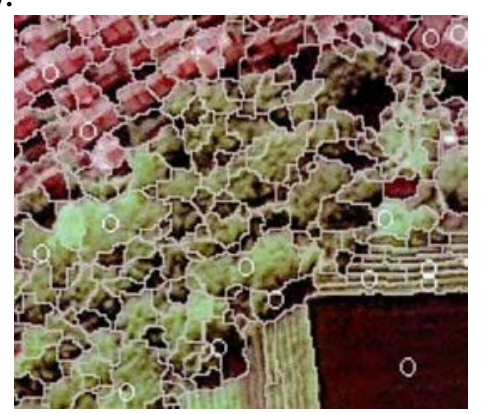

Fig. 1 Remote sensing image segmentation with selected samples

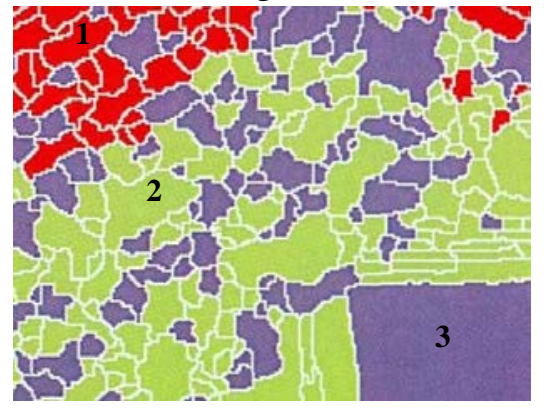

Fig. 2 Preliminary classification result of remote sensing image

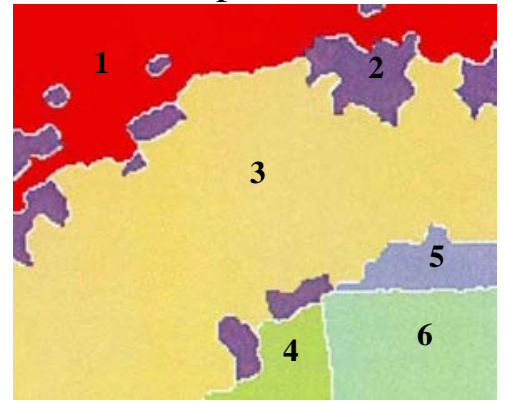

Fig. 3 Final classification result of remote sensing image

To evaluate classification result of remote sensing image based on decision table, a total of 100 unclassified samples are selected from six target earth objects including building, woodland, grassland, water surface, farmland and shadow. The overall accuracy and Kappa's coefficient of the classification results are 0.9125 and 0.9022 respectively.

\section{Conclusion}

In this study, we firstly illustrate the basic ideas and the realization of the classification algorithm in great detail. Then we further verify the classification results by experiments. The proposed classification algorithm can accurately classify the sample objects and reflect the actual distribution of the target earth objects. Our results can be used in monitoring of land cover; monitoring of natural disasters including mud-rock flow, flood and earthquake; military purposes including dynamic monitoring of military target, forces disposition and battlefield information.

\section{Acknowledgement}

In this paper, the research was sponsored by Program for Liaoning hundred million talent project (2014921042).

\section{References}

[1] Xie Miao-miao, Wang Yang-lin, Fu Mei-chen, Zhang Ding-xuan. Pattern Dynamics of Thermal-environment Effect During Urbanization: A Case Study in Shenzhen City, China[J]. Chinese Geographical Science,2013,23(1):101-112. 
[2] Sungwoong K, Chang D Y, Sebastian N, Pushmeet K. Image Segmentation Using Higher-Order Correlation Clustering[J]. IEEE Transactions on Pattern Analysis and Machine Intelligence,2014, 36(9):1761-1774.

[3] Xifa Duan,Zheng Tian,Mingtao Ding, Registration of remote-sensing images using robust weighted kernel principal component analysis[J].International Journal of Electronics and Communications,2013,67:20-28.

[4] Tarabalka Y, Rana A. Graph-cut-based model for spectral-spatial classification of hyperspectral images[C].Geoscience and Remote Sensing Symposium,2014.3418-3421.

[5] Tarabalka,Benediktsson J A,Chanussot J. Multiple spectral-spatial classification approach for hyperspectral data[J]. IEEE Transactions on Geoscience and Remote Sensing,2010,48(11): 4122-4132.

[6] Forestier G, Wemmert C, Puissant A. Coastal image interpretation using background knowledge and semantics[J]. Computers and Geosciences,2013,54:88-96.

[7] Kharol, S.K., Kaskaoutis,D., Badarinath, K. Influence of land use/land cover (LULC) changes on atmospheric dynamics over the arid region of Rajasthan state, India[J]. Journal of Arid Environments,2013,88:90-101.

[8] Kevin Tansey. Object-oriented classification of very high resolution airborne imagery for the extraction of hedgerows and field margin cover in agricultural areas[J]. Applied Geography,2009,29:145-157. 